\title{
A Dual Target-Directed Single Domain-Based Fusion Protein Against Interleukin-6 Receptor Decelerate Experimental Arthritis Progression Via Modulating JNK Expression
}

\section{Xiaole Chen}

Fujian Medical University

Yize Bian

Fujian Medical University

Yongqing Xie

Fujian Medical University

Ningning Zheng

Fujian Medical University

Kaimei Nie

Fujian Medical University

Rui Liu

Fujian medical University

Mengru Yan

Fujian Medical University

Hongbin Luo

Fujian Medical University

He Wang

Fujian Medical University

Juhua Yang

Fujian Medical University

Nanwen Zhang ( $\sim$ znw@fjmu.edu.cn )

Fujian Medical University

\section{Research Article}

Keywords: Interleukin 6 receptor, Fusion Protein, Prokaryotic expression system, Rheumatoid Arthritis, Inflammatory Cytokine

Posted Date: February 19th, 2021 
DOI: https://doi.org/10.21203/rs.3.rs-212152/v1

License: (c) (1) This work is licensed under a Creative Commons Attribution 4.0 International License. Read Full License

Version of Record: A version of this preprint was published at Inflammation on March 10th, 2021. See the published version at https://doi.org/10.1007/s10753-021-01446-7. 


\section{Abstract}

The currently used anti-cytokine therapeutic antibodies cannot selectively neutralize pathogenic cytokine signalling that cause collateral damage to protective signalling cascades. The single domain chain firstly discovered in Camelidae displays fully functional ability in antigen-binding against variable targets, which has been seemed as attractive candidates for the next-generation biologic drug study. In this study, we established a simple prokaryotic expression system for a dual target-directed single domain-based Fusion Protein against the interleukin- 6 receptor and human serum, albumin, the recombinant anti-IL-6R fusion protein (VHH-0031). VHH-0031 exhibited potent anti-inflammatory effects produced by LPS on cell RAW264.7, where the major cytokines and NO production were down-regulated after $24 \mathrm{hr}$ incubation with $\mathrm{VHH}-0031$ in a dose-dependent manner. In vivo, $\mathrm{VHH}-0031$ presented significant effects on the degree reduction of joint swelling in the adjuvant-induced arthritis (AIA) rat, having a healthier appearance compared to the dexamethasone. The expression level of JNK protein in the $\mathrm{VHH}-0031$ group was significantly decreased, demonstrating that $\mathrm{VHH}-0031$ provides a low-cost and desirable effect in the treatment of more widely patients.

\section{Introduction}

Rheumatoid arthritis (RA), characterized by swelling, autoantibody production and bone destruction, is a chronic autoimmune inflammatory disease [1]. The increasing number of RA patients and their families are suffering a substantial burden related to socioeconomic costs, progressive disability, and even early death [2]. A series of continuously developing therapeutic solutions are used in clinical trials against RA, including non-steroidal anti-inflammatory drugs, disease-modifying ani-rheumatic drugs (DMARDs) of synthetic origin and of biological origin [1,3]. However, the conventional drugs present low effectiveness and side effects that requires the researchers to seek new therapeutic substitutes.

The critical damage of RA is widely studied and well known, though the physiochemical, genetic, and environmental factors involved in RA are so complicated that the specific underlying mechanism remains unknown. The bioactivities of major inflammatory cytokines in the pathogenesis of RA has been understood. Interleukin-6 (IL-6), a pro-inflammatory and immunoregulatory cytokinin, plays critical roles in an array of biological processes, where dysregulation and overproduction of IL- 6 presents a considerable role in the rheumatoid arthritis development [4]. Tocilizumab, an anti-IL-6R monoclonal antibody, is successfully employed in the therapy of RA [5]. However, besides the alleviation effects on the RA disease symptoms, tocilizumab presents serious side effects, such as acute pyelonephritis, parotid glands, and inflammation of the upper respiratory system as well [6]. Such currently used therapeutic antibodies are difficult for them to separate pathogenic signalling from physiological signalling [7]. Furthermore, the high cost, short half-life, and application of these biologics limit their clinical efficacy and offer unwanted side effects that require researchers to develop better solutions for RA.

Single domain antibodies, referred to as $\mathrm{VHH}$ or nanobodies, constructed only by the natural camel variable domain of heavy-chain, display numerous superior features, such as low molecular weight and 
immunogenicity, highly affinity to all potential targets [8]. Besides these, it is simple to link two or three single domain chains in a single polypeptide chain by utilizing the genetic engineering method, which can be used to bind one or two even more targets at that same time [7, 9-10]. To overcome the expression limitation, we simplified the expression and the purification procedures of a dual target-directed single domain-based Fusion Protein against the interleukin-6 receptor and human serum albumin ( $\mathrm{VHH}-0031)$. The constructed recombinant anti-IL-6R fusion proteins by linking 2 single domain chains against the proinflammatory cytokine receptor(IL-6R)and human serum albumin (HSA) respectively [11-12]. The single domain chains against HSA have been reported to not only significantly increase the half-life of antibodies in vivo, but also selectively direct the antibodies to specific organs in order to express biofunctions [13-14]. The present study aimed to explore the therapeutic effects of the recombinant antiIL-6R fusion proteins (VHH-0031), obtained by our developed prokaryotic expression system, on adjuvantinduced arthritis (AIA) rats, and elucidate its underlying mechanism of inflammatory mediator or IL-6R in rheumatoid arthritis.

\section{Methods And Materials \\ 2.1 Strains and vectors}

The pET28a + plasmid and E. coli BL21(DE3) strain (Beijing Quanshijin Biotechnology Company) were used for the overexpression of the recombinant anti-IL-6R fusion protein (VHH-0031).

\subsection{Construction of overexpression plasmids}

The cDNA encoding the recombinant anti-IL-6R fusion protein, $\mathrm{VHH}-0031$, using the sequence information published in patent US2012/0093839 and further modified by software Codon usage database(http://www.kazusa.or.jp/codon), JCATロhttp://www.jcat.de】and DNAワwork http://mcl1.ncifcrf.gov/dnaworks $\square$, was made as a synthetic gene (Generay Biotech Co., Ltd) obtained the restrictive sites of $\mathrm{Ncol}$ and Xhol. It was cloned in framework through multiple cloning sites linked to pET28a+. At the $3^{\prime}$-end of the gene with a plasmid $6 \times$ His tag. The construct was verified by sequencing.

\subsection{The preparation of the recombinant anti-IL-6R fusion proteins}

The 100ng plasmid of pET28a-the recombinant anti-IL-6R fusion protein designed and constructed by our lab was transformed into competent cell, BL21(DE3), (Beijing Quanshijin Biotechnology Company) by the heat shock method. Single colonies were selected and resuspended in $5 \mathrm{ml} \mathrm{TGE} \mathrm{buffer,} \mathrm{then} \mathrm{ultrasonic}$ crushed on the ice for $30 \mathrm{~min}$. The recombinant anti-IL-6R fusion proteins fused with His tag were expressed as insoluble inclusion bodies in E.coli and purified, followed by a BeaverBeadsTM IDA-Nickel protocol (Suzhou Beaver Bioengineering Company). The purified proteins were desalted with Äkta (GE Healthcare, USA) and analyzed by $10 \%$ SDS-PAGE.

\subsection{Cell proliferation assay}


RAW 264.7 cell lines were inoculated at a density of $5 \times 10^{3} / \mathrm{mL}$ onto 96 -well plates. It was purchased from the Shanghai Institute of Cellular Biology of the Chinese Academy of Science (Shanghai, China), and cultured with different concentrations of the recombinant anti-IL-6R fusion protein for $24 \mathrm{~h}$. Add 3(4,5-dimethylthiazol-2-yl)-2,5-diphenyl- tetrazolium bromide (MTT) to each well with a final concentration of $0.5 \mathrm{mg} / \mathrm{mL}$, and incubate for 4 hour at $37^{\circ} \mathrm{C}$, add DMSO to dissolve the formazan crystals. Then check the absorbance at a wavelength of $570 \mathrm{~nm}$.

\subsection{Determination of TNF- $a$, IL-1 $\beta$, IL- 6 and NO production}

ELISA was used to detect the production of proinflammatory cytokines TNF-a, IL-1 $\beta$, and IL- 6 in the cell culture. The culture medium was collected and centrifuged at $12000 \mathrm{rpm}$ for $10 \mathrm{~min}$. The supernatants were collected and used to detect TNF- $a$, IL-1 $\beta$, and IL- 6 levels with ELISA kits, according to the manufacturer's protocol. The Griess assay was performed to detect NO [15].

\subsection{Construction of the rat model of AlA model}

Forty Sprague-Dawley rats were selected, 8 of which were randomly selected as the control group. The rats were given intradermal injections of $0.1 \mathrm{~mL}$ normal saline in the left posterior paw pad. The other 32 rats were injected with the same volume of Freund's Complete Adjuvant (FCA) at the same location to establish the AIA rat. This was designated as day 0 . AIA animals were randomly divided into four groups on the 7th day: (1) AIA model group, (2)Dexamethasone (DEX) group, (AIA + intravenous administration of $0.2 \mathrm{mg} / \mathrm{kg}$ DEX per three day), (3) high dose of the recombinant anti-IL-6R fusion protein group (AIA + intravenous administration of $0.5 \mathrm{mg} / \mathrm{kg} \mathrm{VHH}-0031$ per three day) and (4) low dose of the recombinant anti-IL-6R fusion protein group (AIA + intravenous administration of $0.1 \mathrm{mg} / \mathrm{kg} \mathrm{VHH-0031} \mathrm{per} \mathrm{three} \mathrm{day).}$ Meanwhile, the control group and the model group were given the same saline intravenously. Clinical evaluations, including standardized arthritis scores and edema measurements, were performed on the day before immunization (baseline) and on the day after starting the medication (post-dosing), at most 36 days. All procedures were performed in accordance with protocols approved by the Ethics Review Committee for Animal Experimentation of Fujian Medical University (No. 2017-052). All animals were raised in the Laboratory Animal Center of Fujian Medical University (Certificate No. SCXK (Fujian) 20160006), where the animal work has taken place and animal handling procedures were performed in strict accordance with the care of laboratory animals, according to the Fujian Province Zoological Society.

\subsection{Hematoxylin-eosin (HE) staining}

The ankle joint tissue was washed with ice-cold saline, fixed with paraformaldehyde (4\%), and embedded in paraffin, which was cut into $5 \mu \mathrm{m}$ slices and stained with $\mathrm{HE}$, then photographed under an optical microscope with a magnification of $20 x$.

\subsection{Western blotting}

After the experiment, the rats were sacrificed for cervical dislocation and the total protein was extracted by lapping articular soft tissue with liquid nitrogen and adding RIPA lysis buffer containing $1 \%$ PMSF (Beyotime, Tianjin, China). Protein separation was performed by polyacrylamide gel electrophoresis: the 
protein was separated by $4 \%$ sodium dodecyl sulfate (SDS) gel on $10 \%$ polyacrylamide gel electrophoresis (1.5mm thick) in accordance with the Laemmli standard procedure, 50 micrograms of protein loaded into SDS-PAGE gel, and then transferred to the PVDF membrane. The membrane was sealed with $5 \%$ milk in a Tris-buffered saline/Tween 20 (TBS-T) for 3 hours, and the antibodies were incubated overnight $\left(4^{\circ} \mathrm{C}\right)$ with JNK $(46 / 54 \mathrm{kDa}$, Cell Signaling Technology, Cat\#9252), or $\beta$-Actin (45 kDa, Cell Signaling Technology, Cat\#4970), and were incubated with the membranes overnight at $4^{\circ} \mathrm{C}$ on a shaker. The membrane was rinsed with phosphate-buffer saline (PBS), and the secondary antibody (HRP) was incubated at room temperature for $2 \mathrm{~h}$. All experiments were repeated three times and the results were repeatable. The membranes were scanned on a $4000 \mathrm{~mm}$ image workstation (Carestream Health, Inc, USA). The Image J software was used for Image Analysis.

\subsection{Immunohistochemical staining}

For immunohistochemistry, 10\% formalin was fixed for 24 hours, the sample was rinsed and embedded in paraffin and cut into $5 \mu \mathrm{m}$ sections. The samples were dewaxed and rinsed with a phosphate buffer saline (PBS) for 3 times, each time for $10 \mathrm{~min}$, and then treated with $3 \%$ hydrogen peroxide for $10 \mathrm{~min}$ at room temperature. After being washed by PBS for $15 \mathrm{~min}$, sections were blocked with $5 \%$ bovine serum albumin (BSA) at room temperature for $1 \mathrm{~h}$ and then incubated with JNK (46/54 kDa, Cell Signaling Technology, Cat\#9252) at $4^{\circ} \mathrm{C}$ for $24 \mathrm{~h}$. After being washed in PBS again, the sections were incubated with secondary antibody horseradish peroxidase (HRP)-conjugated goat anti-mouse for $45 \mathrm{~min}$, and then cultured with diaminobenzidine. The primary was replaced by PBS as a negative control. Place it under a microscope and select a representative image for analysis.

\subsection{Statistical analysis}

All data are expressed as means \pm SD. One-way ANOVA followed by Bonferroni's Multiple Comparison Test, using GraphPad 5.0 software, was used for comparisons among experiment groups. A p-value of less than 0.05 was considered statistically significant.

\section{Results}

\subsection{The preparation of the recombinant anti-IL-6R fusion protein}

The cDNA encoding the recombinant anti-IL-6R fusion protein, VHH-0031, was made synthetically based on the sequence information of patent US2012/0093839 and was further modified by the method that was mentioned previously. (Fig. 1A). The presence of the recombinant protein in the induced E. coli cells showed a molecular weight of $26 \mathrm{kDa}$ on SDS-PAGE and confirmed by a western blot using His-tag specific antibody. Based on SDS-PAGE, the highly expressed VHH-0031 were in the form of insoluble inclusion bodies, but none in the soluble fraction. The inclusion bodies of $\mathrm{VHH}-0031$ were purified by BeaverBeadsTM IDA-Nickel and followed by being refolded at $4^{\circ} \mathrm{C}$ by 20 -fold fresh distilled deionized 
water dilution. As shown in Fig. 1C, the purity of the recombinant anti-IL-6R fusion proteins can achieve $95 \%$.

\subsection{Effect of the recombinant anti-IL-6R fusion proteins on cytokine production by LPS-induced RAW 264.7 cells.}

The results in Fig. 2A presented that VHH-0031 displayed no action on the cellular activity of RAW264.7 cells at a given concentration, demonstrating that $\mathrm{VHH}-0031$ had no cytotoxic effects on RAW264.7 cells at $0-100 \mu \mathrm{g} \cdot \mathrm{mL}^{-1}$. After LPS induction for $4 \mathrm{~h}$, the levels of TNF-a, IL-1 $1 \beta, \mathrm{IL}-6$, and NO significantly elevated, compared to those of the control group $(p<0.001)$. The recombinant anti-IL-6R fusion protein meaningfully reversed the LPS-induced TNF-a, IL-1 $\beta$, IL-6, and NO levels, with $p<0.05$ or $p<0.01$ for the $100,20,4$, and $0.8 \mu \mathrm{g} / \mathrm{ml}$ treatments (Fig. 2B-C).

\subsection{Effect of the recombinant anti-IL-6R fusion proteins on the swelling of ankle joint in AIA rats}

The AIA rats exhibiting significant RA symptoms, including high arthritis scores and paw swelling, was successfully established. After the administration of the recombinant anti-IL-6R fusion proteins, arthritis scores degraded (Fig. 3A) in AIA rats compared to the control group, while the paw swelling of AIA rats was also relieved in a dose-dependent manner (Fig. 3B). The high dose of VHH-0031 (0.5mg/kg) displayed similar amelioration effects to the classical anti-inflammatory glucocorticoids, Dexamethasone (DEX), though the weight loss decreased significantly lower than that of DEX (Fig. 3C).

\subsection{Effect of the recombinant anti-IL-6R fusion proteins on the inflammatory factors level of AIA rats}

As shown in Fig. 3E-F, compared to the control group, the expression level of IL-6, IL-1 3 , and TNF- $\alpha$ in the AIA rats was obviously enhanced with significant difference $(p<0.001)$. After the treatment of DEX $0.2 \mathrm{mg} / \mathrm{kg}$ and the different doses of recombinant anti-IL-6R fusion proteins, VHH-0031, the level of inflammatory factors decreased to indifferent extents with statistical significance $(p<0.05, p<0.01$ or $p<$ $0.001)$.

3.5 Effect of the recombinant anti-IL-6R fusion proteins on the morphological changes of ankle joints in AlA rats

The main pathological features of arthritis are vasospasm formation, synovial hyperplasia, inflammatory cell infiltration, and cartilage destruction. Therefore, we isolated the ankle joints of rats and made sections for histological staining to observe the therapeutic effects of the recombinant anti-IL-6R fusion protein, $\mathrm{VHH}-0031$. As shown in Fig. 4G, in the control group, the sacroiliac joint structure was intact, and the space between the joint cavities was normal: no synovial hyperplasia was seen, no synovial inflammatory cell infiltration was seen, and the articular cartilage structure was intact and clear (10x). In the model group, the structure of the sacroiliac joint was disordered, including the narrow joint space, the 
synovial of the joint hyperplasia, synovial inflammatory cell infiltration. Inflammatory cell infiltration and vasospasm formation were observed in the Dex group $(0.2 \mathrm{mg} / \mathrm{kg})$. A small amount of inflammatory cell infiltration and synovial hyperplasia were observed in the high-dose $\mathrm{VHH}-0031 \mathrm{group}(0.5 \mathrm{mg} / \mathrm{kg})$, and the formation of vasospasm was significantly improved, while a great deal of inflammatory cells infiltrated in the low-dose group VHH-0031 $(0.1 \mathrm{mg} / \mathrm{kg})$, and the joint structure was incomplete and obvious.

3.6 Effects of the recombinant anti-IL-6R fusion proteins on the expression of JNK protein in articular soft tissues of AIA rats

The immunohistochemical staining and western blot results demonstrated that the JNK protein expression in articular soft tissues was dramatically elevated in the AIA model group, compared with the control group. After the treatments of the recombinant anti-IL-6R fusion proteins, the expression level of JNK protein was obviously reduced in a dose manner (Fig. 4A-B), whereas the JNK in DEX group was presented at a high level. The high dose of $\mathrm{VHH}-0031$ exerted the most prominent inhibitory effect of JNK protein (Fig. 4C-D).

\section{Discussion}

The research on the clinical treatment of Rheumatoid arthritis (RA) has been an attracting interest in the recent years, even though it is still difficult to treat all patients in complete remission $[2,16]$. Interleukin- 6 (IL-6) referred as a prominent activator of the acute phase response with extensive biological activities [2, 17]. Meanwhile, the concentration of IL-6 is significantly elevated in the plasma when the level of TNF- $a$ is meaningfully decreased, along with presenting a longer plasma half-life. Thus IL- 6 has been commonly promised as a valuable biomarker to evaluate the therapeutic response and the prognosis of RA. And IL- 6 has been used as potent therapeutic target in clinical treatment as well [18]. Tocilizumab, approved by the FDA for the RA treatment, is a fully humanized anti-IL-6 receptor antibody that can neutralize IL- 6 activity by binding to both the membrane bound IL-6R and the soluble IL- 6 receptor [19]. However, as other drugs used to treat RA, Tocilizumab displays no effects on some patients in the clinical trial, even with unwanted side-effects, since such currently-used anti-IL-6R antibodies are difficult for them to separate pathogenic signalling from physiological signalling $[7,20]$. Furthermore, the obtainment and quality-test of antibodies are complicated that the clinical used therapies are expensive. Therefore, a more costeffective anti-IL-6R therapeutic antibody selectively binds with related cognate antigens in the particular organ are emergency required.

The single domain chain from camelid specific heavy-chain antibodies have been seemed as the nextgeneration anti-cytokine therapies [8, 21-23]. In this study, a dual target-directed single domain-based Fusion Protein against the Interleukin-6 Receptor and Human Serum Albumin, the recombinant anti-IL-6R fusion proteins ( $\mathrm{VHH}-0031)$, were successfully constructed by genetic engineering methods. The $\mathrm{VHH}-$ 0031 obtained by our simplified prokaryotic expression system exhibited a potent anti-inflammatory effect on the LPS-induced RAW264.7 cell. NO and the inflammatory mediator TNF-a, IL-1 $\beta$, and IL-6 production (elicited by LPS stimulation) of RAW264.7 were down-regulated after a $24 \mathrm{hr}$ incubation with 
$\mathrm{VHH}-0031$ in a dose-dependent manner. The recombinant anti-IL-6R fusion protein has presented its extensive anti-inflammatory bioactivities in vitro. Hereby, a simple prokaryotic expression system for large-scale expression, purification, and the refolding of $\mathrm{VHH}-0031$ from inclusion bodies has been established, which can cut down massive production fees.

The adjuvant-induced arthritis (AIA) rats were established to evaluate the activities of VHH-0031 under the rheumatoid arthritis experimental condition in vivo, $\mathrm{VHH}-0031$ can effectively reduce the degree of joint swelling. Furthermore, compared with the traditional anti-inflammatory drugs, Dexamethasone, the high-dose $\mathrm{VHH}-0031(0.5 \mathrm{mg} / \mathrm{kg})$ presented similar therapeutic effects on the treatment of rheumatoid arthritis in the AIA rats, but had less side effects. The appearances of AIA rats, treated by VHH-0031, displayed much healthier than that of the DEX treated group, the weight losses are meaningfully slower as well. VHH-0031 is supposed to be selectively delivered to inflamed tissues or cell lineage by its part of anti-HSA single domain chain, which is assumed to have less side effects. The major inflammatory factors and pannus in the ankle joint were significantly decreased by $\mathrm{VHH}-0031$.

The results of immunohistochemical staining and western blot both exhibited that the expression level of JNK protein in the VHH-0031 treated group was obviously reduced, compared to the increased JNK levels in the RA rat model and DEX-treated group. This demonstrated that $\mathrm{VHH}-0031$ reduce the activation of JNK protein expression, which plays anti-inflammation roles through JNK signalling pathways. Various studies have reported that c-Jun N-terminal kinase (JNK), belonging to Mitogen-activated protein kinases (MAPKs), can regulate the transcription of a series of inducible genes [24]. More precisely, JNK has functions in cell proliferation and apoptosis by stimulating transcription factors and regulating apoptosis-associated genes expression including inflammatory cytokine genes. However, there is little evidence regarding the relationship between IL- 6 and JNK in RA. Previous studies reported that the production of IL- 6 mRNA after LPS stimulation can be selectively regulated by the JNK transduction pathway, where the inhibition of JNK can downregulate the IL-6 expression that may be beneficial for the inflammatory and autoimmune diseases treatment, though only for higher responding patients mediated by the unbalanced Th1/Th17 type of immune response[24]. In our study, the dual target-directed single domain-based fusion protein, $\mathrm{VHH}-0031$, can reduce the release of inflammatory mediators by downregulating the expression of JNK, which can provide a low-cost and desirable effect in treating a wider range of patient.

\section{Declarations}

\section{Ethics approval and consent to participate}

All procedures were performed in accordance with protocols approved by the Ethics Review Committee for Animal Experimentation of Fujian Medical University (No. 2017-052). All animals were raised in the Laboratory Animal Center of Fujian Medical University (Certificate No. SCXK (Fujian) 2016-0006), where the animal work has taken place and animal handling procedures were performed in strict accordance with the care of laboratory animals, according to the Fujian Province Zoological Society. 


\section{Consent for publication}

The manuscript is approved by all authors for publication.

Availability of data and materials

The datasets used and/or analyzed during the current study are available from the corresponding author on reasonable request.

\section{Competing interests}

The authors declare that they have no conflict of interest.

Funding This work was supported by National Natural Science Foundation, China (Grant No. 81402842), The outstanding Talents Training Project of the Educational Office of Fujian Province, China囚Grant No. 2017052区, Fujian provincial health technology project $₫$ China (Grant No. 2018-ZQN-61, 2018-ZQN-64) , Natural Science Foundation of Fujian Province, China (Grant No. 2019J01306ه2020J01634), The Emergency Project on New Coronavirus Pneumonia Prevention Research of Fujian Medical University (Grant No. 2020YJ004) and the Joint Funds for the Innovation of Science and Technology, Fujian Province [Grant No. 2019Y9111].

Author information

Xiaole Chen and Yize Bian contributed equally to this work

\section{Affiliations}

Department of Bioengineering and Biopharmaceutics, School of Pharmacy, Fujian Medical University,Fuzhou City, 350122 Fujian Province, China

Xiaole Chen,Yongqing Xie,Kaimei Nie,Mengru Yan,Rui Liu \& Juhua Yang

Department of Pharmacology, School of Pharmacy, Fujian Medical University, Fuzhou City, 350122 Fujian Province, China

Yize Bian, Ningning Zheng \&Nanwen Zhang

The First Affiliated Hospital of Fujian Medical University,Fuzhou City, 350005 Fujian Province, China Hongbin Luo

School of Integrative Medicine, Fujian University of Traditional Chinese Medicine,Fuzhou City, 350122 Fujian Province, China

He Wang 


\section{Authors' contributions}

Conceptualization, J.Y. and X.C.; methodology, Y.X. and K.N; software, R.L. and M.Y.; validation,H.W.; writing-original draft preparation,N.Z. and Y.B.; funding acquisition, N.Z.,X.C. and H.L.All authors read and approved the final manuscript.

\section{Corresponding author}

Correspondence to Nanwen Zhang, He Wang or Juhua Yang.

\section{Acknowledgements}

The authors wish to thank Fujian Medical University for providing their equipment to conduct the experiment.

\section{References}

[1] S. Sung, D. Kwon, E. Um, B. Kim, Could Polyphenols Help in the Control of Rheumatoid Arthritis?, Molecules (Basel, Switzerland), 24 (2019)1589.

[2] J.A. Sparks, Rheumatoid Arthritis, Annals of internal medicine, 170 (2019) Itc1-itc16.

[3] H. Yu, R. Zeng, Y. Lin, X. Li, S. Tasneem, Z. Yang, Y.X. Qiu, B. Li, Y.H. Wang, X. Cai, W. Wang, Kadsura heteroclita stem suppresses the onset and progression of adjuvant-induced arthritis in rats, Phytomedicine : international journal of phytotherapy and phytopharmacology, 58 (2019) 152876.

[4] Kang S, Tanaka T, Narazaki M, Kishimoto T. Targeting Interleukin-6 Signaling in Clinic. Immunity. 50(2019)1007-23.

[5] J.D. Alves, A. Marinho, M.J. Serra, Tocilizumab: is there life beyond anti-TNF blockade?, International journal of clinical practice, 65 (2011) 508-513.

[6] J.M. Kremer, R. Blanco, M. Brzosko, R. Burgos-Vargas, A.M. Halland, E. Vernon, P. Ambs, R. Fleischmann, Tocilizumab inhibits structural joint damage in rheumatoid arthritis patients with inadequate responses to methotrexate: results from the double-blind treatment phase of a randomized placebo-controlled trial of tocilizumab safety and prevention of structural joint damage at one year, Arthritis and rheumatism, 63 (2011) 609-621.

[7] M.A. Nosenko, K.N. Atretkhany, V.V. Mokhonov, G.A. Efımov, A.A. Kruglov, S.V. Tillib, M.S. Drutskaya, S.A. Nedospasov, VHH-Based Bispecific Antibodies Targeting Cytokine Production, Frontiers in immunology, 8 (2017) 1073.

[8] Liu M, Li L, Jin D, Liu Y. Nanobody-A versatile tool for cancer diagnosis and therapeutics. Wiley interdisciplinary reviews Nanomedicine nanobiotechnology(2021):e1697. 
[9] S. Chen, J. Li, Q. Li, Z. Wang, Bispecific antibodies in cancer immunotherapy, Human vaccines \& immunotherapeutics, 12 (2016) 2491-2500.

[10] M.W. Fanger, P.M. Morganelli, P.M. Guyre, Bispecific antibodies, Critical reviews in immunology, 12 (1992) 101-124.

[11] K. Conner, C. Pastuskovas, M. Soto, V. Thomas, M. Wagner, D.J.m. Rock, Preclinical characterization of the ADME properties of a surrogate anti-IL-36R monoclonal antibody antagonist in mouse serum and tissues, mAbs, 12 (2020) 1746520.

[12] M. Van Roy, C. Ververken, E. Beirnaert, S. Hoefman, J. Kolkman, M. Vierboom, E. Breedveld, B. t Hart, S. Poelmans, L. Bontinck, A. Hemeryck, S. Jacobs, J. Baumeister, H. Ulrichts, The preclinical pharmacology of the high affinity anti-IL-6R Nanobody ${ }^{\circledR}$ ALX-0061 supports its clinical development in rheumatoid arthritis, Arthritis research \& therapy, 17 (2015) 135.

[13] R.E. Kontermann, Dual targeting strategies with bispecific antibodies, mAbs, 4 (2012) 182-197.

[14] K. Coppieters, T. Dreier, K. Silence, H. de Haard, M. Lauwereys, P. Casteels, E. Beirnaert, H. Jonckheere, C. Van de Wiele, L. Staelens, J. Hostens, H. Revets, E. Remaut, D. Elewaut, P. Rottiers, Formatted anti-tumor necrosis factor alpha $\mathrm{VHH}$ proteins derived from camelids show superior potency and targeting to inflamed joints in a murine model of collagen-induced arthritis, Arthritis and rheumatism, 54 (2006) 18561866.

[15] L. Schmölz, M. Wallert, S. Lorkowski, Optimized incubation regime for nitric oxide measurements in murine macrophages using the Griess assay, Journal of immunological methods, 449 (2017) 68-70.

[16] I. Colmegna, B.R. Ohata, H.A. Menard, Current understanding of rheumatoid arthritis therapy, Clinical pharmacology and therapeutics, 91 (2012) 607-620.

[17] X.L. Chen, Y. Wang, W.W. Peng, Y.J. Zheng, T.N. Zhang, P.J. Wang, J.D. Huang, Q.Y. Zeng, Effects of interleukin- 6 and IL-6/AMPK signaling pathway on mitochondrial biogenesis and astrocytes viability under experimental septic condition, International immunopharmacology, 59 (2018) 287-294.

[18] J. Schroeder, K. Ross, K. McIntosh, S. Jabber, S. Woods, J. Crowe, J. Patterson Kane, J. Alexander, C. Lawrence, R. Plevin, Novel protective role for MAP kinase phosphatase 2 in inflammatory arthritis, RMD open, 5 (2019) e000711.

[19] J. Scheller, E. Engelowski, J.M. Moll, D.M. Floss, Immunoreceptor Engineering and Synthetic Cytokine Signaling for Therapeutics, Trends in immunology, 40 (2019) 258-272.

[20] R.M. Fleischmann, A.M. Halland, M. Brzosko, R. Burgos-Vargas, C. Mela, E. Vernon, J.M. Kremer, Tocilizumab inhibits structural joint damage and improves physical function in patients with rheumatoid arthritis and inadequate responses to methotrexate: LITHE study 2-year results, The Journal of rheumatology, 40 (2013) 113-126. 
[21] Y. Kim, H. Yi, H. Jung, Y.A. Rim, N. Park, J. Kim, S.M. Jung, S.H. Park, Y.W. Park, J.H. Ju, A Dual Targetdirected Agent against Interleukin-6 Receptor and Tumor Necrosis Factor a ameliorates experimental arthritis, Scientific reports, 6 (2016) 20150.

[22] M. Arbabi-Ghahroudi, Camelid Single-Domain Antibodies: Historical Perspective and Future Outlook, Frontiers in immunology, 8 (2017) 1589.

[23] A. Takayanagi, T. Miyakawa, A. Asano, J. Ohtsuka, M. Tanokura, M. Arioka, Expression, purification, refolding, and enzymatic characterization of two secretory phospholipases $A \nabla$ from Neurospora crassa, Protein expression and purification, 115 (2015) 69-75.

[24] C.H. Shang, Q.Q. Zhang, J.H. Zhou, Oridonin Inhibits Cell Proliferation and Induces Apoptosis in Rheumatoid Arthritis Fibroblast-Like Synoviocytes, Inflammation, 39 (2016) 873-880.

\section{Figures}

Fig.1

A

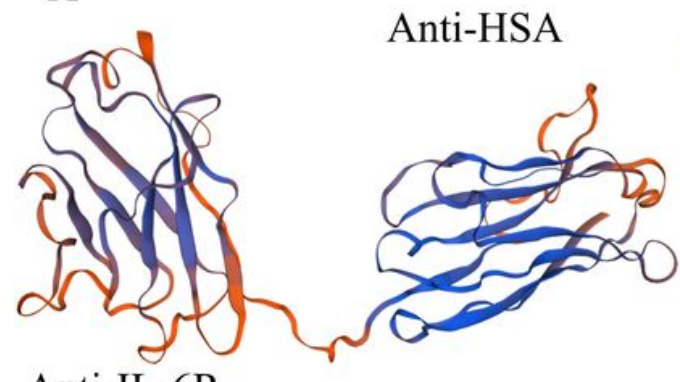

Anti-IL-6R
B

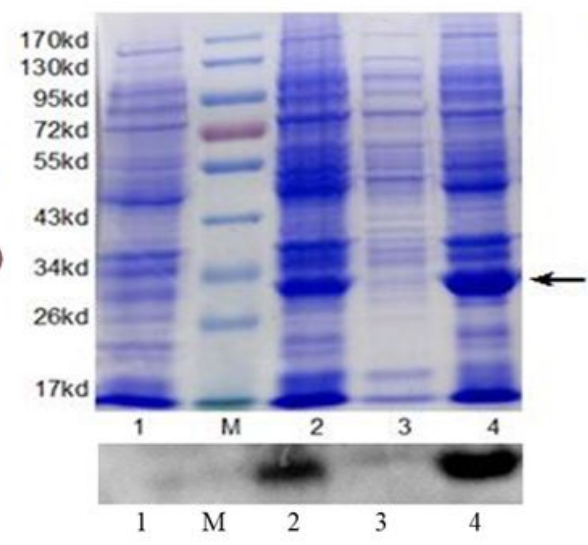

$\mathrm{C}$

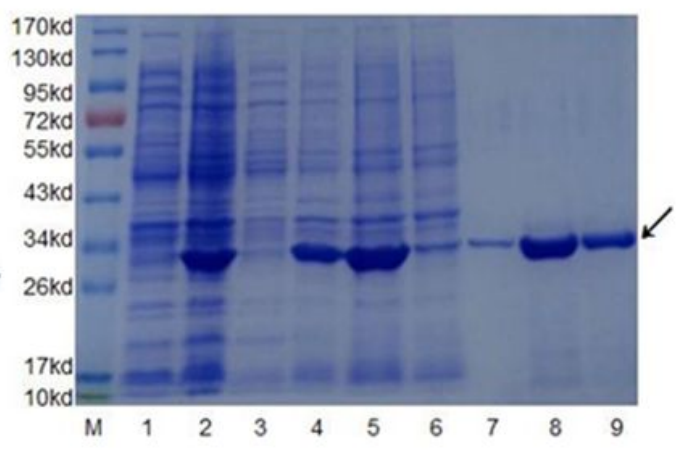

\section{Figure 1}

The preparation of the recombinant anti-IL-6R fusion protein, VHH-0031. (A) 3D structure of VHH-0031 mimicked by SWISS-MODEL. (B) Identification of VHH-0031 by SDS-PAGE and western blot. 1: uninduced, 2: IPTG-induced, 3: supernatant, 4: sediment, M: protein ladder. (C) Identification of the purity of VHH0031 purified by IDA-Nickel with His-tag using Coomassie brilliant blue staining. 1: Induced 
BL21(DE3)/VHH-0031; 2: sediment; 3: supernatant; 4-6: washed IBs; 7-8: renaturation protein; 9: further purified protein by IDA-Nickel.

Fig.2

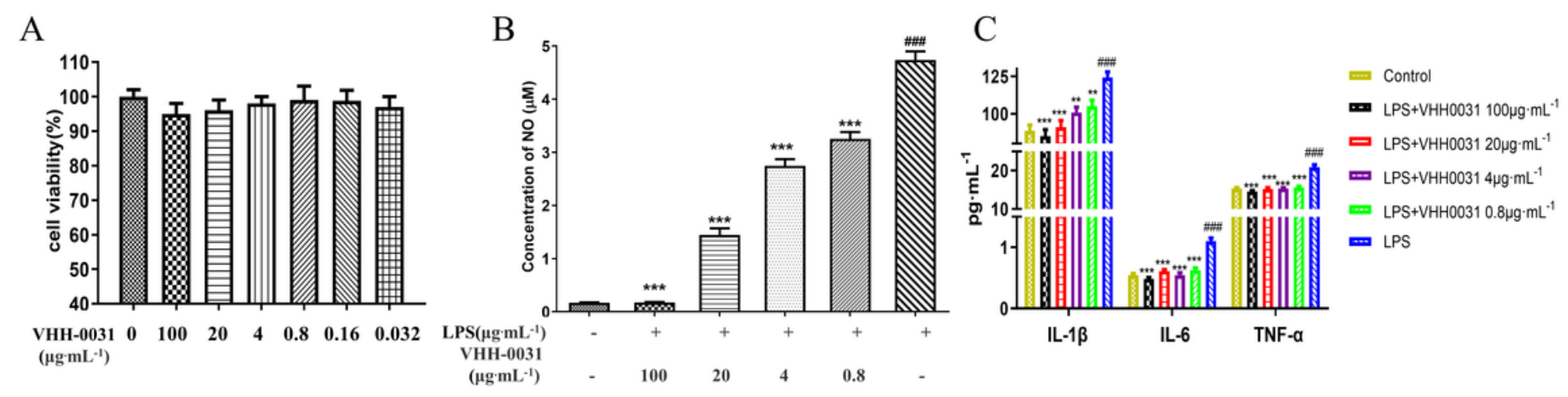

\section{Figure 2}

The inhibitory effects of the recombinant anti-IL-6R fusion protein on inflammatory mediators in vitro. (A)The inhibitory effects of VHH-0031 on the cell viability of RAW 264.7 cells. (B) The inhibitory effects of VHH-0031 on NO production and (C) inflammatory mediators induced by LPS in RAW 264.7 cells. RAW 264.7 cells were incubated with or without LPS for $4 \mathrm{~h}$ and then treated with or without VHH-0031(100 $\mu \mathrm{g} / \mathrm{ml}, 20 \mu \mathrm{g} / \mathrm{ml}, 4 \mu \mathrm{g} / \mathrm{ml}, 0.8 \mu \mathrm{g} / \mathrm{ml}$ ) or PBS (as a negative control). Error bars represent data from three independent experiments. \#\#\#p<0.001 vs control, ${ }^{\star *} \mathrm{p}<0.001 \mathrm{vs}$ LPS group. 
Fig.3
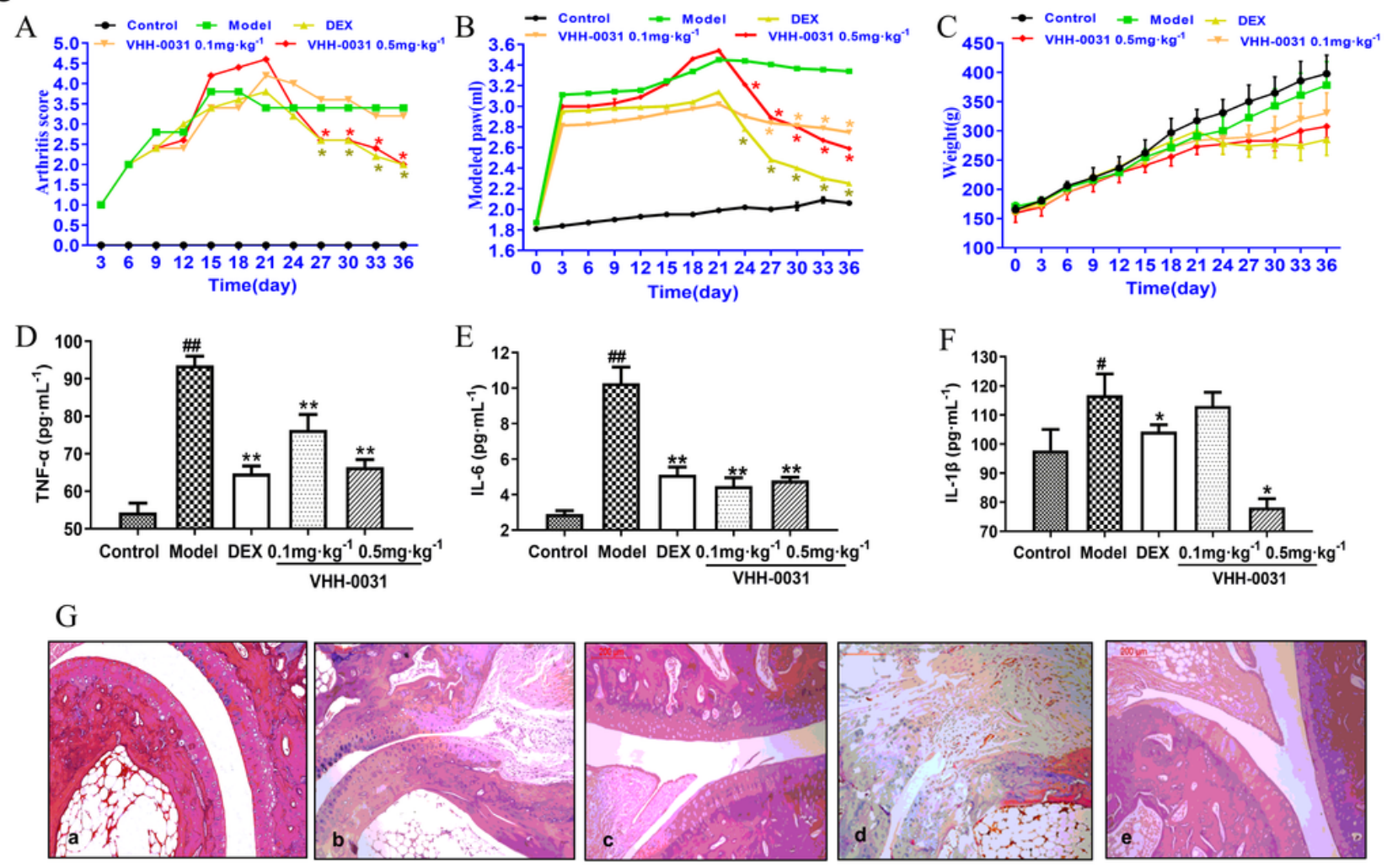

\section{Figure 3}

The amelioration effect of the recombinant anti-IL-6R fusion protein in AIA rats. (A) Arthritis severity score (B) Paw swelling and (C) weight loss of control and AIA rats injected with DEX and VHH-0031. Effect of the recombinant anti-IL-6R fusion protein on inflammatory mediators in AIA rats. (D) TNF-a囚(E) IL-6, (F) IL$1 \beta$, (G) Pathological changes of ankle joint in AIA rats, (a)control group, (b) model group, (c)DEX group , (d) $\mathrm{VHH}-0031(0.1 \mathrm{mg} / \mathrm{kg})$ and $(\mathrm{e}) \mathrm{VHH}-0031(0.5 \mathrm{mg} / \mathrm{kg})$. Sections were stained with H\&E.\#\#p<0.01 vs control, ${ }^{\star} p<0.05,{ }^{\star *} p<0.01$, ${ }^{\star \star *} p<0.001$ vs model, 
Fig.4

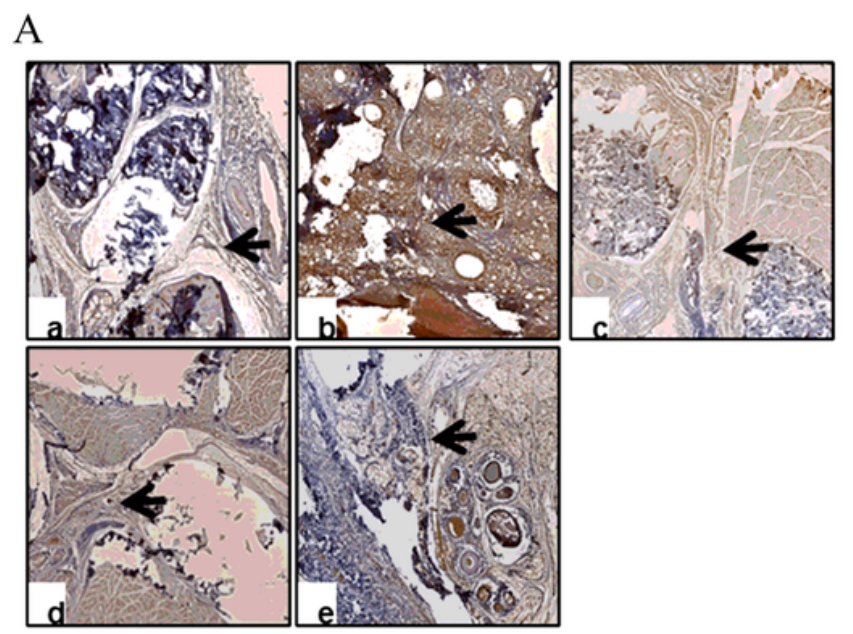

B

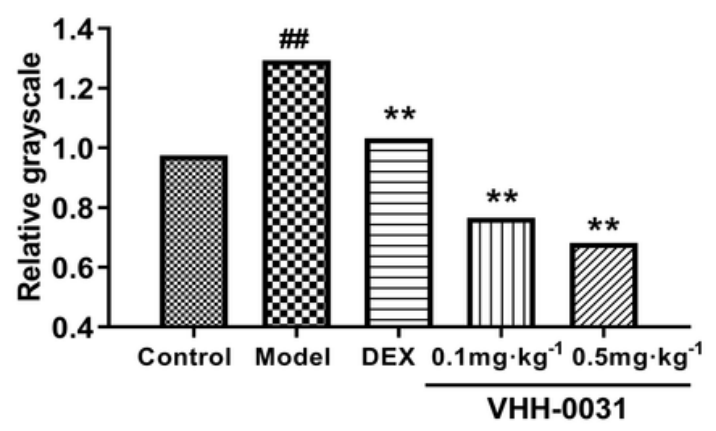

C

D

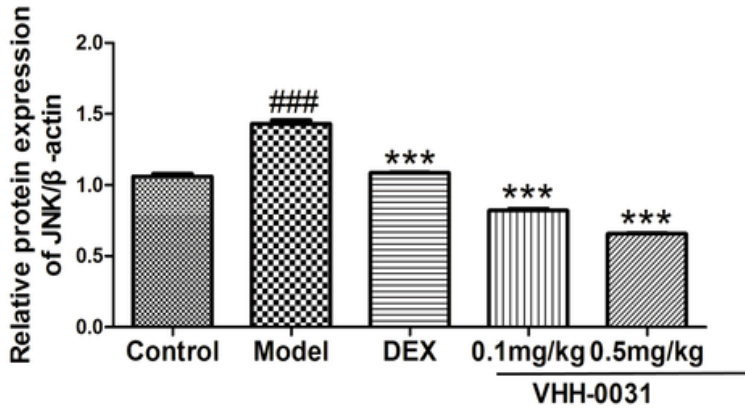

\section{Figure 4}

Effects of the recombinant anti-IL-6R fusion protein on JNK signal pathways in AIA rats. The AIA rats were treated as described in Fig.4(A-B) Representative images of immunohistochemical staining of JNK on modelling foots inflammatory factors in AIA rats. (C-D) Expression of JNK protein levels determined and measured by western blot. Data are presented as the Mean $\pm S E M$. \#\#\#p<0.001vs control, ${ }^{\star \star *} p<0.001$ vs model.

\section{Supplementary Files}

This is a list of supplementary files associated with this preprint. Click to download.

- JNKforFig4C.jpg

- renamed19d94.pdf

- renamed6a4a3.jpg 